\title{
Brain network dynamics underlying visuospatial judgment: an FMRI connectivity study.
}

Citation for published version (APA):

de Graaf, T. A., Roebroeck, A. F., Goebel, R., \& Sack, A. T. (2010). Brain network dynamics underlying visuospatial judgment: an FMRI connectivity study. Journal of Cognitive Neuroscience, 22(9), 2012-2026. https://doi.org/10.1162/jocn.2009.21345

Document status and date:

Published: 01/09/2010

DOI:

10.1162/jocn.2009.21345

Document Version:

Publisher's PDF, also known as Version of record

Document license:

Taverne

Please check the document version of this publication:

- A submitted manuscript is the version of the article upon submission and before peer-review. There can be important differences between the submitted version and the official published version of record.

People interested in the research are advised to contact the author for the final version of the publication, or visit the DOI to the publisher's website.

- The final author version and the galley proof are versions of the publication after peer review.

- The final published version features the final layout of the paper including the volume, issue and page numbers.

Link to publication

\footnotetext{
General rights rights.

- You may freely distribute the URL identifying the publication in the public portal. please follow below link for the End User Agreement:

www.umlib.nl/taverne-license

Take down policy

If you believe that this document breaches copyright please contact us at:

repository@maastrichtuniversity.nl

providing details and we will investigate your claim.
}

Copyright and moral rights for the publications made accessible in the public portal are retained by the authors and/or other copyright owners and it is a condition of accessing publications that users recognise and abide by the legal requirements associated with these

- Users may download and print one copy of any publication from the public portal for the purpose of private study or research.

- You may not further distribute the material or use it for any profit-making activity or commercial gain

If the publication is distributed under the terms of Article $25 \mathrm{fa}$ of the Dutch Copyright Act, indicated by the "Taverne" license above, 
See discussions, stats, and author profiles for this publication at: https://www.researchgate.net/publication/26869902

\section{Brain Network Dynamics Underlying Visuospatial Judgment: An fMRI Connectivity Study}

Article in Journal of Cognitive Neuroscience · October 2009

DOI: 10.1162/jocn.2009.21345 · Source: PubMed

CITATIONS

37

4 authors, including:

Alard Roebroeck

Maastricht University

144 PUBLICATIONS 5,606 CITATIONS

SEE PROFILE

Alexander T Sack

Maastricht University

222 PUBLICATIONS 6,968 CITATIONS

SEE PROFILE

Some of the authors of this publication are also working on these related projects:

Project $\quad$ EuSNN - European School of Network Neuroscience View project

Project Developmental and task-dependent plasticity in speech perception View project
READS

182

Rainer Goebel

Maastricht University

528 PUBLICATIONS 30,883 CITATIONS

SEE PROFILE 


\title{
Brain Network Dynamics Underlying Visuospatial Judgment: An fMRI Connectivity Study
}

\author{
Tom A. de Graaf ${ }^{1,2}$, Alard Roebroeck ${ }^{1,2}$, Rainer Goebel ${ }^{1,2}$, \\ and Alexander T. Sack ${ }^{1,2}$
}

\begin{abstract}
Previous functional imaging research has consistently indicated involvement of bilateral fronto-parietal networks during the execution of visuospatial tasks. Studies with TMS have suggested that the right hemispheric network, but not the left, is functionally relevant for visuospatial judgments. However, very little is still known about the interactions within these fronto-parietal networks underlying visuospatial processing. In the current study, we investigated task modulation of functional connectivity (instantaneous correlations of regional time courses), and task-specific effective connectivity (direction of influences), within the right fronto-parietal network activated during visuospatial judgments. Ten healthy volunteers performed a behaviorally controlled visuospatial judgment task (ANGLE) or a control task (COLOR) in an fMRI experiment. Visuospatial task-specific activations were found in posterior parietal cortex (PPC) and middle/inferior frontal gyrus (MFG). Functional connectivity within this network was task-
\end{abstract}

\section{INTRODUCTION}

Visuospatial processing refers to the spatial perception, recognition, and analysis of visual input. Visuospatial judgment is one example of higher-order visuospatial processing. It can involve the analysis of spatial features of visual stimuli such as distances, angles, or more generally spatial relations, between stimulus parts or aspects of visual images. In visuospatial judgment such analysis is, by definition, goal-related. It therefore often involves a focus on certain spatial features that are relevant to a particular task or goal. Visuospatial judgment is essential to human interaction with the environment.

To understand its neural correlate in the brain, extensive research has been done using functional neuroimaging methods such as positron emission tomography (Mellet et al., 1996; Haxby et al., 1991) and functional magnetic resonance imaging (fMRI; Goebel, Linden, Lanfermann, Zanella, \& Singer, 1998; Cohen et al., 1996), both providing measures of metabolic activity in restricted brain regions. This research has revealed network correlates of visuospatial tasks (Trojano et al., 2000; Goebel

\footnotetext{
${ }^{1}$ Maastricht University, The Netherlands, ${ }^{2}$ Maastricht Brain Imaging Center, Maastricht, The Netherlands
}

modulated, with significantly higher connectivity between PPC and MFG during ANGLE than during COLOR. Effective connectivity analysis for directed influence revealed that visuospatial taskspecific projections within this network were predominantly in a frontal-to-parietal direction. Moreover, ANGLE-specific influences from thalamic nuclei to PPC were identified. Exploratory effective connectivity analysis revealed that closely neighboring clusters, within visuospatial regions, were differentially involved in the network. These neighboring clusters had opposite effective connectivity patterns to other nodes of the fronto-parietal network. Our data thus reveal that visuospatial judgments are supported by massive fronto-parietal backprojections, thalamo-parietal influence, and multiple stages, or loops, of information flow within the visuospatial network. We speculate on possible functional contributions of the various network nodes and informational loops in a neurocognitive model. et al., 1998), visuospatial imagery (Sack, Camprodon, Pascual-Leone, \& Goebel, 2005; Formisano et al., 2002; Trojano et al., 2002; Mellet et al., 1996), visuospatial attention (Luks, Sun, Dale, Miller, \& Simpson, 2008; Giessing, Fink, Rosler, \& Thiel, 2007; Husain \& Nachev, 2007; Mayer, Seidenberg, Dorflinger, \& Rao, 2004; Corbetta, Kincade, \& Shulman, 2002), visuospatial working memory (Klingberg, 2006; Scherf, Sweeney, \& Luna, 2006; Klingberg, Forssberg, \& Westerberg, 2002; Postle, Berger, Taich, \& D'Esposito, 2000; Petit, Courtney, Ungerleider, \& Haxby, 1998), and visuomotor control (Culham, Cavina-Pratesi, \& Singhal, 2006; de Lange, Hagoort, \& Toni, 2005). All of these different functional imaging studies converge to the conclusion that the execution of various visuospatial tasks consistently activates bilateral frontal and parietal regions in the "visuospatial network" of the brain.

However, research using TMS to disrupt regional neural processing has indicated that only the right, not the left, parietal network is functionally relevant for (visuo)spatial processing (Collignon et al., 2008; Valero-Cabre, PascualLeone, \& Rushmore, 2008), visuospatial attention (Jin \& Hilgetag, 2008; Rounis, Yarrow, \& Rothwell, 2007; Kim et al., 2005; Muri et al., 2002; Hilgetag, Theoret, \& PascualLeone, 2001), visuomotor processing (Bestmann, Thilo, 
Sauner, Siebner, \& Rothwell, 2002; Rushworth, Ellison, \& Walsh, 2001), visuospatial imagery (Sack et al., 2005), and visuospatial judgment (Sack et al., 2002, 2007).

This apparent right hemispheric dominance for visuospatial functions was further elucidated by a recent simultaneous TMS and fMRI study of our group, in which the neural correlates of TMS-induced behavioral impairment on a visuospatial judgment task were assessed "on-line" (Sack et al., 2007). To this end, we administered TMS over either left or right posterior parietal cortex (PPC), while concurrently measuring both the behavioral as well as neural consequences of parietal TMS using whole-brain fMRI scanning. Only right parietal TMS, not left, induced a behavioral impairment on the visuospatial judgment task, but not on a control task. Simultaneously, only right parietal TMS, not left, resulted in a TMS-induced BOLD signal decrease throughout a right hemispheric frontoparietal network during visuospatial judgment, but not during control task execution. In other words, TMS over right parietal cortex not only reduced brain activity at the site of stimulation, but also in ipsilateral remote frontal regions, including right $\mathrm{MFG}$. Interestingly, the amount of BOLD signal decrease in this network, both in local and remote regions, correlated highly with the amount of TMS-induced visuospatial impairment.

Taken together, all of these findings seem to indicate that a fronto-parietal network in the right hemisphere underlies visuospatial processing. Moreover, as the most recent simultaneous TMS and fMRI study on visuospatial judgment (Sack et al., 2007) suggested, the interactions within this network are such that disruption of processing at one node of the network (i.e., the parietal node) has measurable and comparable neural effects at the other nodes (e.g., within MFG).

Still, the exact task-specific dynamics, temporal characteristics, and direction of influences within this specific right hemispheric fronto-parietal network during the execution of visuospatial judgments remain unknown. In the current study, we used time-resolved, fast eventrelated fMRI in combination with multivariate data-driven functional and effective brain connectivity analysis tools in order to investigate the brain network dynamics underlying visuospatial judgment in the right hemisphere. We subsequently interpreted these system-level interactions on the cognitive level to construct a neurocognitive model underlying visuospatial judgment.

For optimal comparability with previous research, we adopted the same behavioral tasks used by Sack et al. (2002, 2007): a visuospatial judgment task (ANGLE) and color judgment control task (COLOR). We specifically evaluated task modulation of functional connectivity within this network, and proceeded to investigate directed influences within this task-specific functionally connected network. We purposely adopted a recently developed exploratory effective connectivity analysis technique (Granger causality mapping [GCM]; see Roebroeck, Formisano, \& Goebel, 2005) to avoid restriction of analysis to predefined regions. This way, we were able to explore in depth the interactions within, and beyond, the conventionally defined visuospatial network in the human right hemisphere.

\section{METHODS \\ Participants}

Ten healthy participants ( 5 men, 8 right-handed) were included in this study (mean age $=23.3$ years; $S D=$ 1.8 years). All had normal or corrected-to-normal vision, no history of neuropsychiatric disorders, and eight had previously participated in fMRI experiments. The experiment was approved by the local ethical committee, written informed consent was obtained before participation. Participants were screened for fMRI experimentation safety and received monetary compensation.

\section{Stimuli and Task}

Participants were presented with visual stimuli inside the MRI scanner. Each stimulus was projected for $300 \mathrm{msec}$ at center fixation. Participants were asked to fixate at all times, aided by a gray fixation cross between stimuli. The stimuli consisted of schematized analogue clocks with yellow rims and two either white or yellow hands (13/ 33 yellow). The hands of the clocks formed different angles, categorized as small or large (13/33 small). All stimuli and fixation crosses were taken from our previous study (Sack et al., 2007). Participants were asked to press one of two buttons per stimulus, depending on whether the stimulus was a target (right index finger response) or a nontarget (right middle finger response). In the ANGLE task, clocks with small angles $\left(30^{\circ}\right.$ or $60^{\circ}$ angles between the hands) were targets, clocks with large angles (bigger than $60^{\circ}$ ) were nontargets. In the COLOR task, clocks with yellow hands were targets, clocks with white hands were nontargets (see Figure 1 for an illustration of stimuli and tasks). Stimuli were presented, and response times recorded, using Presentation Software (Neurobehavioral Systems, San Francisco, CA). Response speed and accuracy were equally emphasized in instructions to the participants. The experimental design was mixed; blocks of stimuli were presented in a rapid event-related design. One block contained 11 trials (10 task trials: 4 targets and 6 nontargets, and one null trial). Throughout a block, the task was constant. The task for each block was made known to the participant prior to the block, in the form of a one-letter cue: "A" for ANGLE, "C" for COLOR. The order of blocks was pseudorandomized, as was the order of trials within the blocks. A total of 28 blocks (=280 task trials), divided equally over two fMRI functional runs, was presented. Within blocks, the intertrial interval was jittered around 3000 to $4500 \mathrm{msec}$. Time between blocks was $7500 \mathrm{msec}$, including the 2000-msec task instruction. 


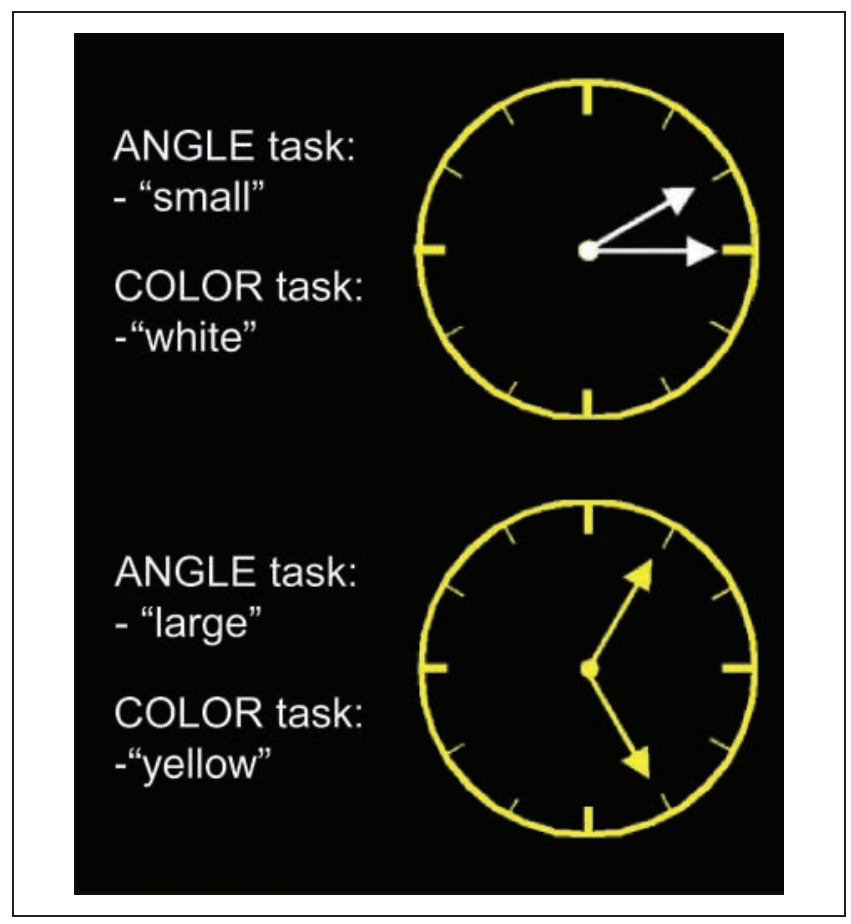

Figure 1. Stimuli and tasks. Two example stimuli are shown with appropriate responses for each task. The same stimuli were used in both the ANGLE and the COLOR tasks. In the ANGLE task, the two response options corresponded to a "small" angle $\left(30^{\circ}\right.$ or $\left.60^{\circ}\right)$ and a "large" angle (more than $60^{\circ}$ ) between the two clock-hands. In the COLOR task, the two response options reflected the two possible clock-hands colors: "yellow" and "white."

\section{MRI Parameters and Functional Data Processing}

MRI was performed using a 3-Tesla Siemens Allegra scanner (Siemens, Erlangen, Germany). A standard transmitreceive head coil was used to obtain high-resolution anatomical [ADNI, T1-weighted, flip angle $(\mathrm{FA})=9^{\circ}, \mathrm{TR}=$ $2250, \mathrm{TE}=2.6 \mathrm{msec}, 192$ slices, field of view $(\mathrm{FoV})=$ $256 \mathrm{~mm}$, isotropic voxel resolution of $1 \times 1 \times 1 \mathrm{~mm}^{3}$ ] and whole-brain functional (T2*-weighted echo-planar imaging; $\mathrm{FA}=60^{\circ}, \mathrm{TR}=1500, \mathrm{TE}=28 \mathrm{msec}$, acquisition gap $=500 \mathrm{msec}, 18$ oblique contiguous slices, slice thickness $=5 \mathrm{~mm}$, FoV $=224 \mathrm{~mm}, 64 \times 64$ voxel matrix, voxel resolution $=3.5 \times 3.5 \times 5 \mathrm{~mm}^{3}$ ) images (for an illustration of slice coverage, see S1 on www.tomdegraaf.com). Participant hearing was protected using ear plugs and headphones. Head movement was restricted using foam pads.

fMRI data were processed using BrainVoyager QX (Brain Innovation, Maastricht, The Netherlands). Preprocessing included interscan slice acquisition time correction, linear trend removal, temporal high-pass filtering to remove lowfrequency drifts, and rigid-body transformation of data to the first acquired image to correct for motion. Please note that functional data were not smoothed spatially; reported data were unsmoothed throughout all analyses. Functional data were coregistered to anatomical data per subject, and subsequently transformed to Talairach space (Talairach and Tournoux, 1988) or aligned to a dynamic group average brain by means of cortex-based alignment (CBA).

\section{Cortex-based Alignment}

For individual right hemispheres, the gray-white matter boundary was determined to segment and reconstruct the cortical surface (Kriegeskorte \& Goebel, 2001). Functional data in volume space were sampled to surface vertices in a direction perpendicular to the gray-white matter boundary, and thus, converted to surface space. The idiosyncratic folding patterns of sulci and gyri were mapped in a spherical coordinate system. In this common space, a reiterative process morphed the individual hemispheric surfaces to a dynamic group average. This resulted in an average group brain. Because functional data were mapped to surface space, the surface-to-sphere and sphere-togroup average transformation mappings also afforded group alignment of functional data. We applied this process of CBA to our general linear model (GLM) contrast analyses and GCM connectivity analyses because it has been shown to yield superior statistical power (Fischl, Sereno, Tootell, \& Dale, 1999).

\section{Activation Data Analysis}

For functional data analysis, BOLD time courses of individual vertices were regressed onto a prespecified model in a conventional GLM. Predictors were based on 300-msec events convolved with a hemodynamic response gamma function (Boynton, Engel, Glover, \& Heeger, 1996). Separate predictors were implemented for ANGLE clock presentations, COLOR clock presentations, and INSTRUCTIONS ("A" and "C" conjoined in one model predictor). Effects of interest included activation during the ANGLE or the COLOR task contrasted with baseline activity and activation during the ANGLE versus the COLOR conditions as contrasted directly in the GLM. Activation differences on the group level were analyzed directly on the vertex level, leading to statistical parametric maps on a group-average brain [an additional patch-of-interest (POI) analysis is presented and discussed on-line: www.tomdegraaf.com]. Corrections for multiple comparisons were made using cluster threshold level estimation (Hagler, Saygin, \& Sereno, 2006; Forman et al., 1995), with 1000 iterations of Monte Carlo simulation setting a statistical threshold of $p<.01$ for the main task effects and $p<.05$ for the ANGLE $>$ COLOR contrast. To ensure generalizability of the results, all analyses reported were performed on a random effects (RFX) level (unless indicated otherwise). BOLD time courses for regions of interest (ROIs) were approximated by means of event-related deconvolution analysis. This method can be applied in rapid event-related designs to extract the BOLD response to single events from the overlapping pattern of activation. Activation is regressed on separate 
"stick" predictors per scanning time point. This results in one beta value per TR in the volumes following an event. These beta values reflect the BOLD response pattern to an event.

\section{Functional and Effective Connectivity Analysis}

Functional connectivity has been defined as correlation between remote neurophysiological events in the temporal domain (e.g., Friston, 1994). Functional connectivity analysis can reveal networks of connected regions that show similar activity changes during mental task performance. Thus, in our case, functional connectivity refers to task-specific instantaneous correlation of BOLD time courses during task execution. Presumably, remote regions with BOLD time courses that correlate during visuospatial judgment task execution, but not execution of a control task, are working together on the mental processes involved in visuospatial judgment. Effective connectivity has been defined as directed influence from one region to another, and allows one to move toward causal inferences (Friston, 1994). More precisely, we examined task-specific effective connectivity to ROIs throughout the brain. To this end, we applied GCM (Roebroeck et al., 2005). GCM requires one to specify a seed region after which measures of (functional or effective) connectivity for all voxels or vertices in the brain are calculated, in reference to the time course in the seeded cluster. The maps obtained illustrate which areas in the brain are functionally connected to the seed region (functional connectivity maps), or which areas in the brain send influence to or receive influence from the seed region (effective connectivity maps).

GCM works on two time series, $X[t]$ and $Y[t]$ (activity over time in Region $X$ and Region $Y$ ). The precise meaning of found connectivity between $X$ and $Y$ is a decrease of uncertainty about time points of $Y$, due to knowledge about past time points of $X$. Simply put, knowing one time course tells you something extra about the other time course. The direction of influence is based on which time course lags behind the other. Thus, if knowing $X[t-1]$ (history of BOLD in Region $X$ ) improves the prediction of $Y[t]$ (BOLD time course of Region $Y$ ) over and above the prediction achieved using $Y[t-1]$ (Region $Y$ 's own BOLD history), then the activity in Region $X$ is said to Granger cause the activity in Region $Y$. By regressing one time series on both itself and another, influences can be inferred with suitable models (vector autoregressive models). Directionality is thus concluded on the basis of temporal precedence. All effective connectivity maps were restricted to vertices that also had high instantaneous correlations to minimize effects of draining veins (for further details, see Roebroeck et al., 2005).

GCM has successfully been applied in previous studies (Bien, Roebroeck, Goebel, \& Sack, 2009; Sack et al., 2008; Sack et al., 2007; Abler et al., 2006). However, these studies only looked at individual participants or mean GCM maps, or merely performed fixed effects GCM analysis. The current study explicitly evaluated statistically thresholded RFX functional and effective connectivity, by calculating RFX GCM maps as follows: (1) GCM maps were calculated for each individual participant in surface space (on the reconstructed cortical surface with functional data projected onto it), or volume space. Statistical testing for individual maps was based on bootstrap simulations (see Roebroeck et al., 2005), with corrections for multiple comparisons based on false discovery rate $(q<$ 0.05) (Genovese, Lazar, \& Nichols, 2002). (2) In surface space, these individual maps were cortex-based aligned, and second-level $t$ tests (per vertex, over subjects) were calculated. Correction for multiple comparisons in these RFX group maps was based on cluster-size threshold estimation (1000 iterations; see Hagler et al., 2006; Forman et al., 1995) (this procedure underlies Figures 3, 4, 5). Alternatively, in volume space, individual maps were overlaid in Talairach space before mean GCM group maps were created (e.g., Figure 6). For a defined threshold, these procedures yield maps of functional or effective connectivity to the seed region-throughout the entire brain. The method thus affords both confirmatory and datadriven exploratory RFX connectivity analysis without prior anatomical and directional constraints, save the specified seed region.

To analyze connectivity in individual participants (and thus create the aforementioned individual GCM maps which were subsequently overlaid to obtain RFX group maps), we determined a POI to seed into the GCM procedure as follows: A GLM conjunction analysis of ANGLE versus baseline and COLOR versus baseline $[(\mathrm{A}>\mathrm{B}) \vee(\mathrm{C}>$ B)] was performed, revealing regions where activity was modulated by both tasks. From these regions, the cluster in PPC with the highest difference in activity between ANGLE and COLOR (with activity higher during ANGLE trials) was determined. This region was assumed to be engaged in visuospatial processing and served as the starting point for GCM analysis of the visuospatial network. Post hoc confirmatory GCM analyses were performed by seeding into the GCM procedure new seed regions defined on the basis of individual GCM maps or group RFX GCM maps.

To summarize, for each individual participant, taskspecific functional connectivity maps were created based on instantaneous correlation of vertices throughout the entire brain, to the seed region. Effective connectivity per experimental condition was calculated in step 2 for each individual participant. In step 3, the individual maps were cortex-based aligned (for the surface space analyses) and overlaid. This created a RFX group GCM map projected onto the average group brain. Statistical significance of the results was tested as described above. Separately, we explored in individual participants, in volume space, the intricate dynamics within the network identified by the conventional GLM contrasts. 


\section{RESULTS}

\section{Behavioral Results}

Reaction times and accuracy were $675.4 \mathrm{msec}(S D=$ 166.0) and 6.3\% incorrect for ANGLE, and $650.5 \mathrm{msec}$ $(S D=175.5)$ and $9.7 \%$ incorrect for COLOR, respectively. Sack et al. (2007) used the same task but found faster average reaction times: $495 \mathrm{msec}$ and $452 \mathrm{msec}$ for ANGLE and COLOR tasks, respectively. But these authors used a block design with regular and predictable stimulus onsets. A brief follow-up behavioral experiment revealed that five new participants were, on average, $156.3 \mathrm{msec}$ quicker to respond in a block design as applied by Sack et al. (2007) than in the mixed design implemented in the current study, confirming experimental design to be responsible for the differences.

\section{Brain Activation Results}

We performed a RFX GLM analysis on the vertex time courses in cortex-based aligned surface space (see Methods). Figure 2A shows thresholded RFX activation maps, on the cortex-based aligned group-average inflated brain,

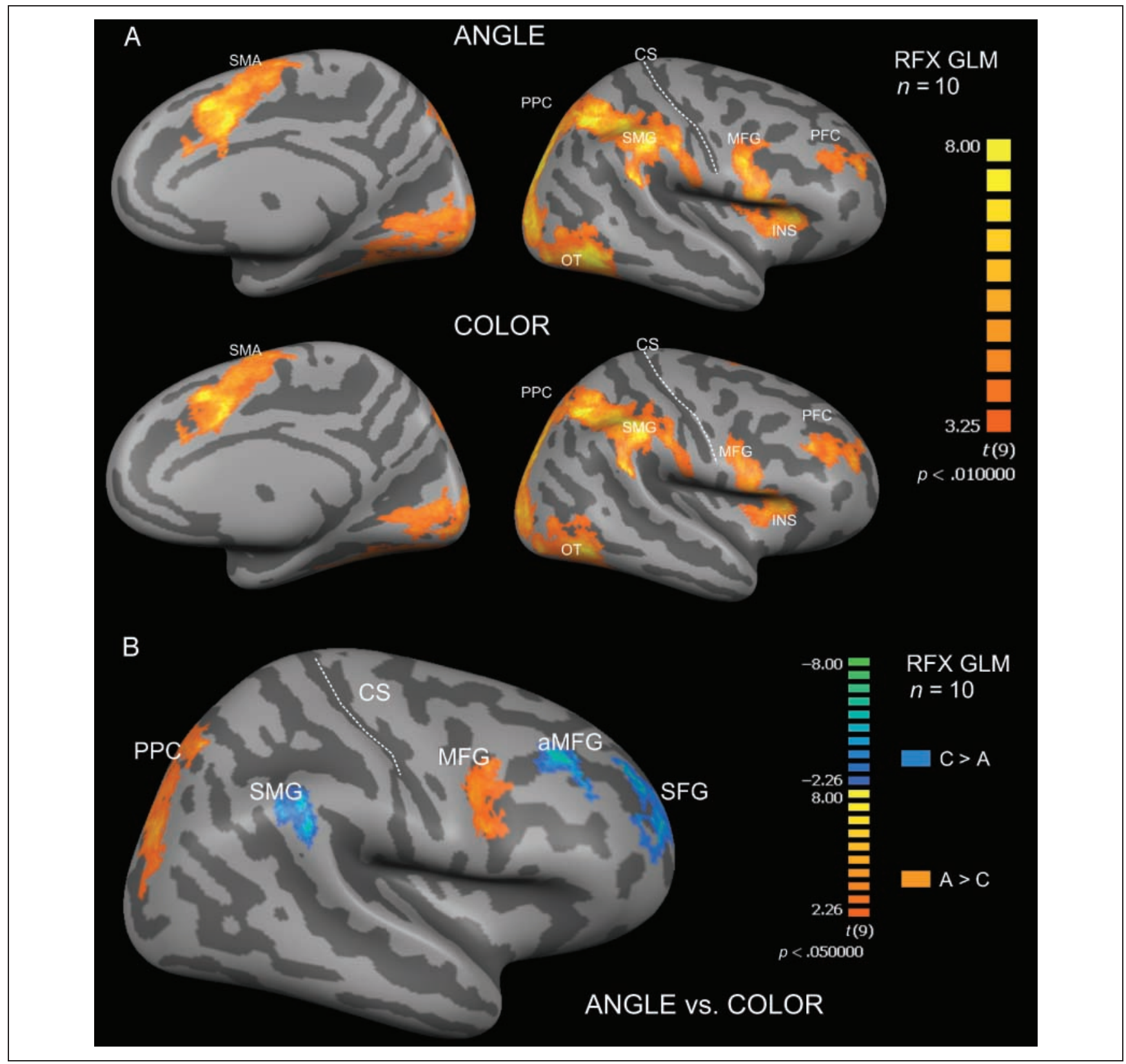

Figure 2. RFX activation maps. (A) Cortex-based aligned RFX GLM for ANGLE and COLOR conditions. Vertices significantly more active during task processing than baseline are shown in orange-yellow $[t(9)=3.25, p<.01$, corrected]. (B) A cortex-based aligned RFX GLM contrast map. Vertices more active during ANGLE are shown in orange-yellow, vertices more active during COLOR are shown in blue-green $[t(9)=2.262$, $p<.05$, corrected] 
for ANGLE versus baseline and for COLOR versus baseline separately $[t(9)=3.25, p<.01$, corrected]. An extensive fronto-parietal activation network is revealed, notably including middle/inferior frontal gyrus (MFG) and PPC. The medial views of the reconstructed right hemisphere reveal activation in supplementary motor area and large regions of the occipital lobe, in both tasks. The lateral views show that regions of prefrontal cortex, occipitaltemporal cortex (OT), and the insula (INS) are activated in both tasks.

To examine which of these activations were privy to the visuospatial network, we contrasted the activity in the ANGLE and COLOR conditions in a RFX GLM. In surface space, after CBA, a RFX GLM on these data revealed two regions to be significantly more active in response to ANGLE executions as compared to COLOR executions (i.e., PPC and MFG), and three regions more active during COLOR as compared to ANGLE [i.e., SMG, an anterior region of MFG (aMFG), and SFG; $t(9)=2.262, p<.05$, corrected]. Figure 2B visualizes these regions in a contrast map (for coordinates and more information on these and other task-specific areas, see www.tomdegraaf.com).

\section{Functional Connectivity during Visuospatial Processing}

Before investigating directionality of influences in the visuospatial network, we examined functional connectivity (instantaneous correlation) within this network during the visuospatial ANGLE task and the COLOR control task. Coactivation of regions during task processing does not guarantee a functional connection, let alone task-specific functional connectivity (instantaneous correlations in regional time courses during one task but not another). However, we were able to demonstrate that PPC engaged in significantly stronger functional connectivity to MFG during visuospatial processing than during our control task. Thus, this fronto-parietal network communicated more vigorously during visuospatial judgment. To visualize the spread of seed regions across participants, Figure 3 shows in varying colors the MFG POIs of all 10 participants (after CBA) seeded into GCM. We computed a RFX group map of task modulation of connectivity, in cortex-based aligned surface space. Figure 3 thus shows which regions were significantly $[t(9)=2.262, p<.05$, corrected] more strongly connected to MFG during ANGLE blocks than during COLOR blocks.

PPC is more connected to MFG, as are parts of parietooccipital cortex, during ANGLE as compared to COLOR pseudoblocks. Thus, this map visualizes statistically significant task modulation of functional connectivity within the visuospatial network. Task modulation is important and informative; it ensures that the results reveal functional brain organization that is task-related. Having established task modulation of the connectivity within the network, we also investigated directionality of influences within this network.

\section{Effective Connectivity during Visuospatial Processing}

RFX GCM effective connectivity analysis, referenced to PPC, revealed a frontal-to-parietal direction of influence. Figure 4 shows statistically significant $[t(9)=2.262, p<$ .05 , corrected] influences to and from the seed region as revealed by GCM, for ANGLE and COLOR separately.

Green areas influence (project to) the PPC seed region (red), whereas blue areas are influenced by PPC. During ANGLE, MFG, a second parietal cluster, and INS all significantly projected to the PPC seed region $[t(9)=2.262$, $p<.05$, corrected]. During COLOR, no statistically significant influences were found. Please note, however, that this lack of statistical significance does not imply that there was absolutely no effective connectivity between PPC and MFG

Figure 3. RFX connectivity difference map. RFX group contrast based on cortex-based aligned functional and anatomical data. This map reveals task modulation of instantaneous functional connectivity between MFG and other parts of the brain. Vertices that are significantly more functionally connected (FC) to MFG during the visuospatial ANGLE task than during the COLOR control task are shown in orange-yellow $[t(9)=2.262, p<.05$,

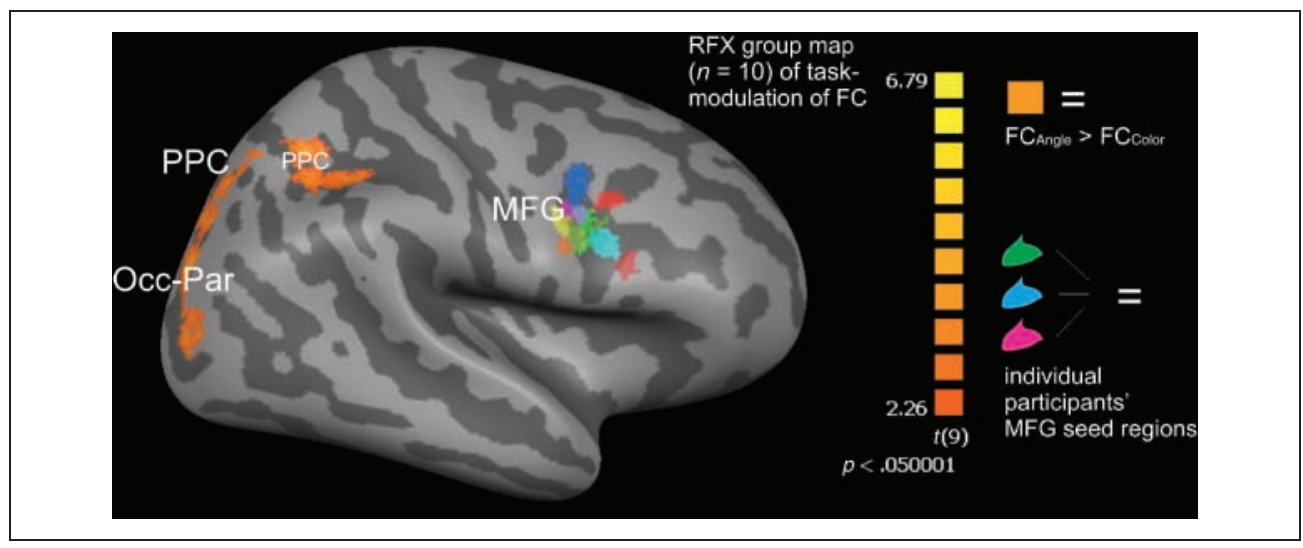
corrected]. Thus, PPC is more connected to MFG during ANGLE than during COLOR. This task modulation of connectivity indicates that the fronto-parietal network operates more as a whole during ANGLE conditions than during COLOR conditions. This group map was calculated after individual functional connectivity maps were obtained, based on individual seed POIs. To illustrate interparticipant spread of these seed regions, the individual MFG POIs on which the analyses were based are shown in varying colors, after cortex-based alignment of the patches. 


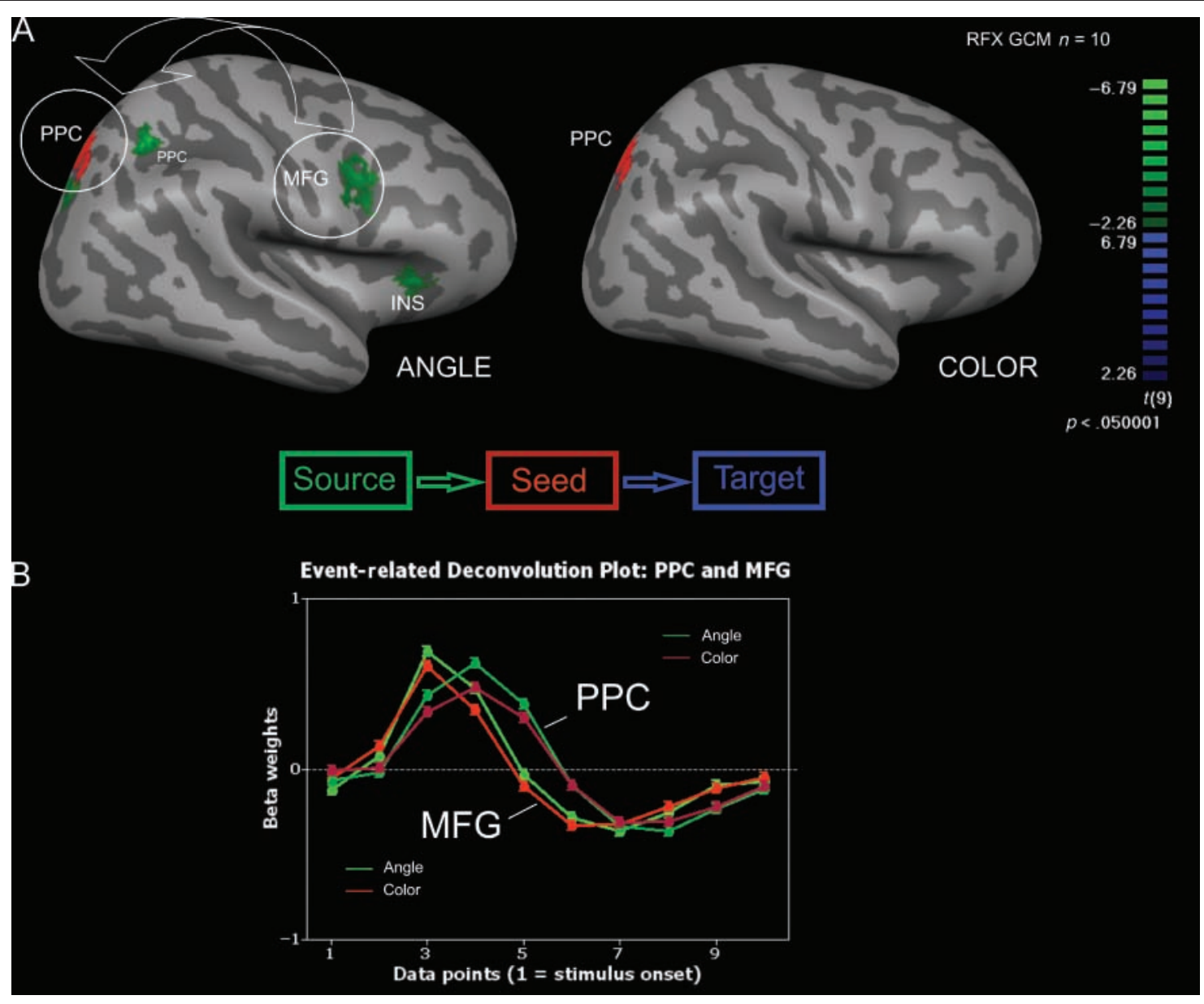

Figure 4. Task-specific effective connectivity. (A) GCMs, showing task-specific directed influences throughout the entire brain, referenced to seed region PPC (in red). Shown here are statistically significant projections $[t(9)=2.262, p<.05$, corrected] to PPC from MFG, INS, and a second parietal cluster, during the ANGLE task specifically. No significant connectivity to PPC was found for the COLOR condition. (B) Event-related deconvolution plots approximate the regional BOLD time courses for PPC and MFG per task. This plot implies earlier involvement of MFG than PPC.

during the COLOR task, but that it was weaker in comparison to the effective connectivity during the ANGLE task and, unlike during ANGLE, not statistically significant (for further illustration of locations and extent of task modulation of effective connectivity, see S2 on www.tomdegraaf. com). A post hoc analysis with seed region MFG confirmed that MFG did significantly project to PPC during ANGLE, but also significantly projected to OT during ANGLE and not during COLOR $[t(9)=2.262, p<.05$, corrected; data not shown]. For PPC and MFG, event-related deconvolution plots approximate the regional time courses. Note that different latencies of the average (deconvolved) BOLD response are not equivalent to GCM directed influences (see Roebroeck et al., 2005, for details). However, in this case, the BOLD time-course plots clearly confirm earlier involvement of MFG than PPC during both tasks, with stronger activation for ANGLE in both regions (see Figure 4).

For several other regions, there were indications of effective connectivity to PPC. Postcentral gyrus (PCG), occipital cortex (OT), and superior frontal sulcus (SFS) were effectively connected to PPC in individual GCM maps, mean GCM group maps (Figure 6), and in the more conservative RFX GCM group analysis, these regions showed a statistical tendency toward PPC during ANGLE $[t(9)=$ $1.83, p<.1$, corrected] but not during COLOR [with the exception of PCG; $t(9)=1.83, p<.1$ ]. Interestingly, SFS showed a particularly strong task modulation of effective connectivity (on par with connectivity modulation in MFG): SFS interacted much more with PPC during ANGLE than during COLOR (see S2 on www.tomdegraaf.com).

To summarize, MFG and INS significantly influenced PPC during ANGLE, but not during COLOR, as indicated by effective connectivity analysis referenced to PPC. Furthermore, there were indications that PCG, OT, and SFS also engage in network interactions during visuospatial processing.

We also identified COLOR-specific regions; aMFG, SFG, and SMG. However, RFX GCM analysis to these regions yielded no significant effective connectivity to these regions in either the ANGLE or the COLOR task, other than vertices or small clusters surrounding the seed region, with the exception of SFG in the ANGLE task, where SFG received 
influence from INS $[t(9)=2.262, p<.05$, corrected; data not shown].

To examine dynamics within the network on the subcortical level, we performed RFX GCM analysis, referenced to the seed region PPC, in volume space. We were able to demonstrate effective connectivity with subcortical structures during visuospatial processing. During both ANGLE and COLOR tasks, but seemingly more strongly during ANGLE, PPC received influence from bilateral thalamic nuclei (see Figure 5A). A follow-up RFX GCM analysis seeded with the right hemispheric thalamic cluster confirmed that this cluster significantly projected to PPC $[t(9)=2.262, p<.05$, uncorrected $]$ during ANGLE, but not during COLOR, as depicted in Figure 5B. Event-related

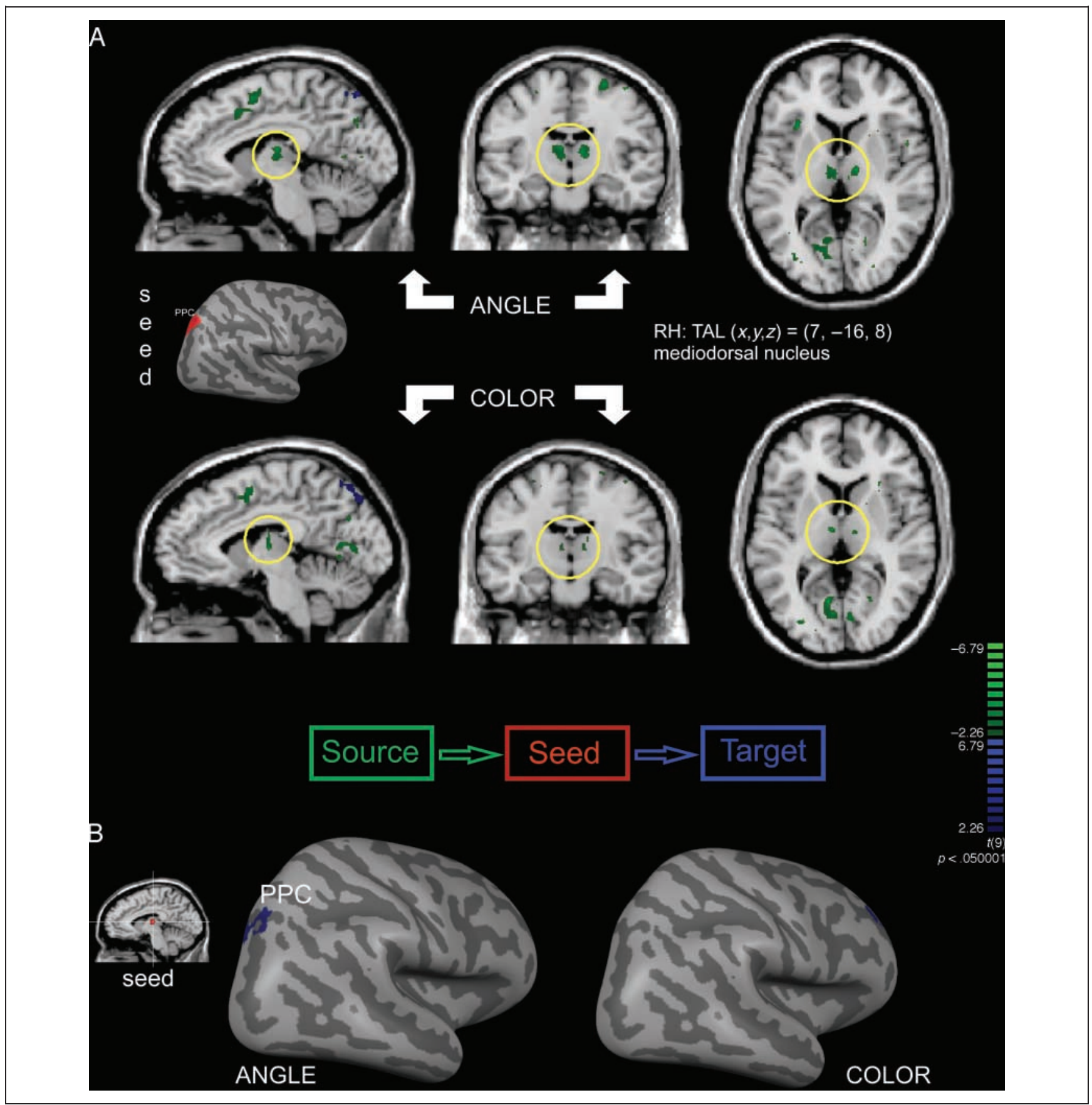

Figure 5. Subcortical involvement. (A) RFX GCM analysis was run in volume space to explore subcortical involvement during visuospatial processing. Bilateral thalamic clusters (green) significantly project to PPC during the ANGLE task, the same seed region shown in A (and in middle inset) $[t(9)=2.262, p<.05$, uncorrected], and seemingly less so during the COLOR control task. Probabilistic mapping suggested these clusters to be the mediodorsal nuclei. (B) Taking right hemispheric thalamic nucleus shown in A as seed region in a RFX GCM analysis in volume space, right hemispheric PPC significantly received influence (receiving areas are in blue) during ANGLE, but not during COLOR $[t(9)=2.262, p<.05$, uncorrected]. This validates the results in (A) and confirms subcortical involvement in visuospatial processing specifically. 
deconvoluted time courses for PPC and right thalamus revealed an earlier BOLD response/peak in right thalamus than in PPC (see S5 on www.tomdegraaf.com).

To constrain the location of the thalamic nuclei, we performed a probabilistic mapping of the individually defined thalamic clusters. The Talairach coordinates of the point of greatest interindividual overlap were $(-6,-16$, 8 ) for the left hemispheric cluster, and $(7,-16,8)$ for the right hemispheric clusters, corresponding to bilateral mediodorsal nuclei.

\section{Complex Communication Dynamics within the Visuospatial Network}

The exploratory character of GCM has the advantage that connectivity analysis is not confined to ROIs as defined in a GLM analysis, except for the seed region. Importantly, this allows the technique to identify interacting regions in the brain that were not identified in a GLM. Additionally, GCM can separate differently interacting regions, which were merged into one activated cluster in a GLM contrast test. Indeed, we found that within ROIs, implicated in visuospatial processing by the GLM analyses, there may be a functional segregation of neighboring clusters in terms of interactions with remote regions. Among these neighboring clusters, a projecting cluster (influencing other regions of the visuospatial network) and a receiving cluster (receiving influence from other regions of the visuospatial network) could be discerned. Such closely neighboring clusters with opposite effective connectivity patterns were found within PPC and OT in all 10 participants, and within MFG in 6 of the participants. Figure 6A gives an example of such neighboring clusters in one representative participant ("NK"), illustrating how anatomically close these clusters were in OT and MFG. The seed region in the GCM analysis underlying this figure (Figure 6A) was PPC in "NK." Such segregation of temporal involvement could not be detected by GLM analysis, which, as we showed in Figure 2, lumps these clusters together in one functional "blob."

In a post hoc analysis, we proceeded to seed these neighboring clusters into individual GCM analyses to confirm the effective connectivity of these clusters. The resulting group mean GCM maps (calculated in volume space and projected onto a partly inflated cortex-based aligned group brain) are displayed in Figure 6B. It is clear from these images that clusters identified as "projecting" only sent influence to regions throughout the brain, whereas clusters identified as "receiving" primarily received influence. Importantly, these visualized interactions are very sensible, including primarily the regions identified in prior GLM and GCM analyses.

From these maps, it seems that the projecting ventral and dorsal stream (OT and PPC) clusters project mainly to each other and neighboring regions, not to frontal regions. Yet the receiving ventral and dorsal stream clusters receive influence from SFS and MFG. The receiving PPC cluster receives influence from INS and prefrontal regions also. Overall, these analyses seem to confirm the flow of information primarily from frontal regions back to the temporal-occipital and parietal regions. Yet, the picture has been enriched by complex dynamics between the larger regions implicated in visuospatial processing, with neighboring clusters differentially involved over time. An important caveat to these interpretations is that the maps presented in Figure 5B are averaged, overlaid GCM maps based on the individual participant analyses. They are thus mean GCM maps; not statistically thresholded on the group level (although thresholded and corrected for multiple comparisons on the individual map level; see Methods). Nevertheless, they unmistakably confirm that the neighboring clusters have opposite projection characteristics. Moreover, the regions either receiving or projecting influence to the seeded clusters are part of the network as already established above, which lends added credibility to the maps as presented here.

Another interesting aspect of these data is that projecting clusters within a region (e.g., OT) seem to send influence to a region (e.g., PPC), which subsequently projects back to the neighboring cluster (in OT) —as illustrated by an overlap of regions that receive influence and project influence in Figure 6 [please find on-line Figure S3: www. tomdegraaf.com, which shows the GCM maps of projecting and receiving clusters (left- and right-side maps in Figure 6) overlaid, to facilitate such comparisons]. The projecting cluster consistently showed an earlier BOLD response/peak, as compared to the receiving cluster, for all participants and all regions (see Figure S4 on-line). Taken together, these findings seem to support a form of recurrent processing underlying our visuospatial judgment task (see Discussion).

To summarize, based on our GCM analyses, we were able to functionally segregate projecting versus receiving clusters in anatomically adjacent brain regions within at least PPC, MFG, and OT. These projecting and receiving clusters may be differentially involved in earlier and later processing stages during the visuospatial judgments. This suggests that nodes of the visuospatial network are engaged in multiple loops. A complex matrix of activation can thus be inferred involving these regions, with multiple distinctive clusters, that as a whole seems to eventually establish the mental performance of the task. Aside from PPC and MFG, contributions from OT, INS, SFS, and PCG were suggested by our GCM results.

\section{DISCUSSION}

Our data replicate earlier studies on visuospatial processing by revealing involvement of frontal and parietal regions during visuospatial judgment. However, our results go beyond previous research by demonstrating that these regions were indeed operating as a network during execution of our task. Thus, we revealed statistically significant 
Figure 6. Differential connectivity patterns for closely neighboring clusters. (A) An example of one representative participant ("NK"); individual GCM analysis in volume space referenced to PPC (shown in red in the middle inset). Green clusters project to PPC, blue clusters receive influence from PPC. Closely neighboring clusters, one of which sent influence to PPC while its neighbor received influence from PPC, were revealed by GCM in various regions implicated in visuospatial processing. (B) RFX mean GCM effective connectivity maps, referenced to either the projecting (green in $\mathrm{A}$ ) or the receiving (blue in A) functional clusters In individual GCM analyses in each participant, projecting and receiving clusters were discerned and fed into GCM analysis as seed regions (red and asterisked on the rendered brains) Subsequently, and displayed here for the angle condition, RFX group maps were obtained for projecting clusters (left column) and receiving clusters (right column) for PPC (top row), MFG (middle row), and OT (bottom row). All maps have equal thresholds. The maps in the left column and the right column have been overlaid in Figure S3 in the supplementary material on-line (www.tomdegraaf.com). Overlap between them indicates, in confirmation of the example given in $\mathrm{A}$, that a form of recurrent processing occurs.

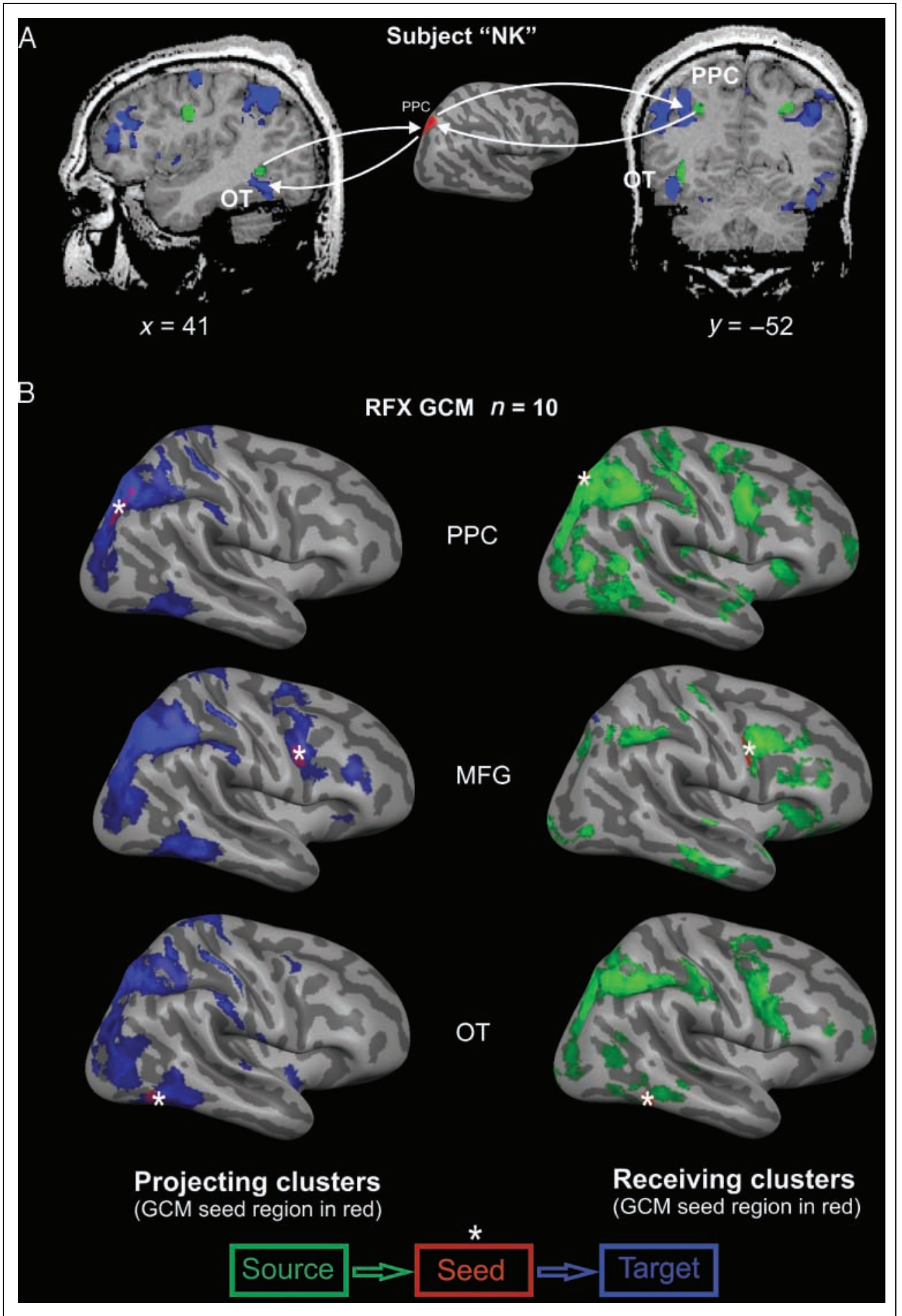

task modulation of functional connectivity within the visuospatial network (i.e., between PPC and MFG). Furthermore, GCM allowed us to investigate directional influences within this network, revealing a frontal-to-parietal information flow, and subcortical involvement that may be task-specific. Moreover, exploratory effective connectivity analysis on an individual participant basis reliably identified neighboring clusters with opposite connectivity patterns to other nodes of the network. Such connectivity-segregated clus- ters were found within the visuospatial areas conventionally lumped together by GLM analysis. This seems to confirm functional segregation of these clusters, and suggests the possibility of multiple communication loops within the visuospatial network. Such results provide new insights into functional brain organization and are generative of new neurocognitive hypotheses. Below, we propose a speculative interpretation of what neurocognitive mechanisms are subserved by the brain events described in this article. 


\section{New Brain Activation Findings}

Our main ANGLE-specific activations, in MFG and PPC, mirror an established body of work on visuospatial cognition (see Introduction). However, in previous research using the exact same stimuli and tasks, our COLOR-specific regions were not consistently identified (Sack et al., 2002, 2007). This may be attributed to several methodological differences between our and previous work that could have lead to respective differences in statistical sensitivity for revealing these COLOR-specific brain regions. First, we applied new procedures of data analysis: Our study involved CBA, GLM analysis in surface space, and no spatial smoothing. Second, whereas the previous work with our stimuli and tasks involved block designs, we employed a rapid event-related design. This resulted in more frequent changes between tasks and in unpredictable delays between trials. As indicated in the Results section and confirmed in a pilot experiment, the new design also resulted in different behavioral results. It is difficult to disentangle these factors. Unfortunately, we gained no further insights from GCM analysis as almost no significant effective connectivity was found to these regions in both tasks. However, the fact that our conservative GCM analysis did not produce miscellaneous influences to these regions further validates the results we did find for the experimental task. The remainder of the discussion will focus on these interactions underlying execution of the ANGLE task.

\section{Fronto-parietal Information Flow}

A primary aim of our study was to examine the nature of interactions between MFG and PPC during the execution of visuospatial judgment. Interestingly, our results revealed information flow to be mainly from MFG and other frontal regions, including INS and possibly SFS, PCG, and OT, toward PPC. Although the projections were not necessarily unidirectional (see below), the dominant frontoparietal direction is noteworthy, because a classical view of visuospatial processing involves a hierarchical dorsal striate-parieto-frontal stream. Dominant MFG-to-PPC projections seem to go against this stream.

One explanation of this direction of influence stems from the nature of our cognitive task. Visuospatial judgment is, by definition, goal-related: A judgment involves options, choices, and, in our case, a template to match input to [the "target angle" is the angle between clockhands that defines the border between Response 1 (small angle) and Response 2 (large angle)]. We propose that MFG was instrumental in a process of stored template matching, biasing the processing of visuospatial features in PPC using top-down signals, in light of the task-relevant comparison with the target angle template. Right MFG has been implicated in various higher-order central executive processes, such as selection, monitoring, and organization of processing (e.g., Curtis \& D'Esposito, 2003; Rypma \&
D’Esposito, 2003; Collette \& Van der Linden, 2002); all forms of top-down (attention) control (see Yeh, Kuo, \& Liu, 2007). Already some years ago, ventral lateral prefrontal cortex was proposed to serve as an early recipient of information from posterior parietal regions and as the site where comparisons are made with representations in working memory (D'Esposito et al., 1998; Owen, Evans \& Petrides, 1996; Petrides, 1994). More recently, directed influences from right MFG to right superior parietal cortex have been found in a flanker task with a spatial twist and in an fMRI study using structural equation modeling to study effective connectivity (Erickson, Ringo Ho, Colcombe \& Kramer, 2005). This information flow was interpreted in terms of attentional set (based on, for example, Banich et al., 2000). Moreover, it has been suggested that neural processing in lower areas is most efficient under the direction of, or in interaction with, top-down signals from a higher region (Kveraga, Ghuman \& Bar, 2007). In sustained attention tasks (which our tasks seem to be), it has been suggested specifically that right medial frontal regions influence, top-down, posterior cortex to bias and enhance processing (e.g., see Sarter, Givens \& Bruno, 2001, for a review). Taken together, this literature leads us to speculate that the MFG-to-PPC projections were focusing the processing in PPC on the relevant visuospatial features, either prior to stimulus onset to sensitize the relevant neurons and/or immediately upon stimulus presentation to bias processing toward the relevant visuospatial information. We return to this interpretation below, when we present our neurocognitive model taking into account the other information flows identified in our study.

Methodological limitations constrain the extent of our interpretation. Unfortunately, bidirectional influences between two regions are lost in GCM maps. Because the effective connectivity values are based on a difference measure of the influence in two opposite directions (for details, see Roebroeck et al., 2005), the results reflect the dominant influence only. Thus, a unidirectional MFG-to-PPC influence identified by GCM does not preclude a communication flow in the reverse direction per se. It just means that, overall, MFG predominantly influenced PPC during ANGLE pseudoblocks. The interpretation of bidirectional, but asymmetrical, fronto-parietal flow is consistent with research by Edin, Klingberg, Stodberg, and Tegner (2007). These authors integrated EEG, fMRI, and neurocomputational modeling, to show that, in a visuospatial working memory task, a bidirectional fronto-parietal model with stronger frontal-to-parietal (i.e., SFS-to-IPS) connections than parietal-to-frontal (i.e., IPS-to-SFS) connections best explained the data. Indeed, in their study, stronger SFSto-IPS effective connectivity was shown to protect against distracters on visuospatial working memory performance. Thus, fronto-parietal asymmetry was functionally relevant. Apparently, this may be a mechanism that is not specific to our task only.

Because GCM is based directly on BOLD signals rather than neural signals, intrinsic hemodynamic response differences 
between brain areas could confound GCM results of a given condition. This is countered by using a control condition (in our case, COLOR) to ensure task specificity of the connectivity results. Our MFG-to-PPC influence was significant only in the visuospatial task and not in the control task. Another limitation of GCM is that it remains uncertain whether information flows directly from an identified region to the seed region, or via other brain areas. In light of this, it becomes important to take into account connectivity results throughout the brain.

\section{System-level Task Performance: Complex Dynamics with Recurrent Processing}

As mentioned, a supposedly unidirectional finding might, in fact, hide a bidirectional communication loop. This limitation was discussed by Roebroeck et al. (2005), alongside a positive note: If bottom-up projections and top-down projections between two regions target neighboring, but anatomically separated, clusters (rather than exactly the same voxels/vertices), then GCM will be ideally suited to separate and detect these functionally distinct clusters which, in conventional fMRI analysis, would be lumped together. We here show this conjecture to be accurate, having identified neighboring clusters with opposite projection characteristics in complex network dynamics. Such neighboring clusters were revealed in several nodes of the network, notably PPC, MFG, and OT. The network correlate of visuospatial processing in our paradigm may, to an extent, operate as a whole, interacting in such a way as to continuously send back and forth neurally coded information, moving toward a "steady state" of the neural network. Such mechanisms could never be recorded by fMRI studies. Alternatively, regions within a closely knit network might still make functionally separable contributions to the overall mental process. Also, there might be a finite set of communication loops within the network, especially a finite number of spatially separated recurrent loops. We here could demonstrate at least two clusters within larger regions (e.g., OT) that were differentially involved. More specifically, it could be hypothesized, based on the patterns of connectivity and the corresponding time courses, that the projecting cluster was involved at an earlier stage than the receiving cluster (for time courses and discussion, see supplementary on-line material, Figure S4).

Recently, early and late fronto-parietal network components were also identified by Sack et al. (2008). In their fMRI study, participants were asked to mentally imagine the assembly of a spatial structure, based on visual or auditory instructions, and finally compare this structure to a visually presented structure. Thus, their task was more extensive than the one adopted in the current study, taking several seconds to complete. However, that task also essentially contained a visuospatial judgment component. These authors identified functionally segregated early and late fronto-parietal network clusters, based on a data-driven functional decomposition analysis called fuzzy clustering. Early versus late components were found in several regions, including posterior parietal, frontal, and occipitaltemporal regions. We here show that "early" (projecting) and "late" (receiving) neighboring clusters could be revealed within broadly the same regions, using a different method of analysis (GCM) during a mentally much more succinct process. Such converging evidence lends bidirectional support for the findings of our two studies. Moreover, it suggests that the multiple loops, or processing stages, were not artifactual to either behavioral task. However, whereas Sack et al. extracted two fronto-parietal networks from the data, our data are supportive of a form of recurrent processing between the major nodes of the network. For instance, the projecting cluster in OT sends influence to the same area in PPC that subsequently sends influence back to OT (but to the neighboring, receiving cluster). The GCM maps on the left and right in Figure 6B overlap in part, but far from completely (see Figure S3 on-line: www.tomdegraaf.com). It thus seems that during our visuospatial task, dynamic, recurrent interactions occur within one, however nonstatic, network. Whereas recurrent processing is a well-researched mechanism in lower-level vision (Lamme \& Roelfsema, 2000), to our knowledge, it has not been demonstrated in such a form as we present here, in higher-order cognition. The question remains what, exactly, the functional relevance of these distinct connectivity loops might be. To better understand this issue, it may help to review the evidence for specific functions associated with the various nodes in our visuospatial network.

\section{Functional Roles of Visuospatial Network Nodes}

PPC seems to be involved in many spatial processes, as has been discussed in the Introduction. It is therefore plausible to assume that the analysis of the spatial features of our stimuli was centered in PPC. Lehmann, Vannini, Wahlund, Almkvist, and Dierks (2006) used task demand, as measured by RT, as a predictor in GLM analysis. Their cognitive task was highly similar to the one used in this study; involving judgment of clock-hands. PPC, a region near MFG, as well as INS, all revealed BOLD signal correlation to task demand: strong evidence for direct visuospatial task involvement of all these regions.

Both SFS and MFG have been implicated in spatial working memory tasks. The role of SFS in spatial working memory seems well established (Zarahn, Aguirre, \& D’Esposito, 1999; Courtney, Petit, Maisog, Ungerleider, \& Haxby, 1998; Petit et al., 1998). To properly perform our task, a representation of the "target angle" must have been maintained in order to serve as template to match new visuospatial input to. The SFS may have stored that template. Sack et al. (2008) instructed participants to imagine a certain visuospatial object, which was rotated compared to a remembered target object. Participants were then asked to determine whether the two objects were the 
same. Using GCM, Sack et al. found a premotor-to-parietal direction of influence underlying this task. On inspection, their premotor area may correspond to our SFS. To actually perform the stored template matching, MFG could come into play, as detailed above. This proposed mechanism is in line with previous research. Glahn et al. (2002) showed involvement of both MFG and SFS in a spatial working memory task, but were able to dissociate the contributions of both regions. SFS was involved specifically in maintenance of spatial working memory contents, whereas MFG seemed to be involved specifically in spatial manipulation of these contents (see also Postle et al., 2000).

Ventral area OT may engage in the recognition and formation of the clock object, which our stimuli comprised. This object information was likely more relevant to the processing of an angle between real object parts: the clock-hands, than a basic property such as color, which is observed at a glance. The involvement of OT in object processing is relatively established (e.g., Grill-Spector, 2003). More difficult to interpret are the results on the thalamo-parietal projections. The mediodorsal nucleus of the thalamus has been regarded as the gateway to prefrontal cortex (e.g., Tanibuchi \& Goldman-Rakic, 2003), rather than parietal cortex. Yet, there were indications in an fMRI investigation in humans that BOLD signals in the mediodorsal nucleus correlated to those in superior parietal cortices during a spatial attention task (Buchsbaum et al., 2006). It is possible that our thalamic nucleus projecting to PPC was not, after all, the mediodorsal nucleus, because reliable specification of thalamic nuclei in human group fMRI studies is difficult due to intersubject variability (Davis, Kwan, Crawley \& Mikulis, 1998), and because direct connections between other thalamic nuclei and parietal cortex have been found in humans (Behrens et al., 2003). Alternatively, the thalamo-parietal influence may have been indirect, which is an option GCM inherently cannot disqualify (Roebroeck et al., 2005). Irrespectively, we propose that the thalamus did project to PPC in a kind of "fast route," albeit direct or indirect (see also Chambers, Payne, Stokes \& Mattingley, 2004). Combined with the data and literature on other regions in our visuospatial network, this leads us to propose the following neurocognitive model (illustrated in Figure 7).

\section{A Proposal for Neurocognitive Mechanisms Underlying Visuospatial Judgment}

Visual information proceeds through the retina to thalamic clusters and on to early visual cortex (occipital cortex). Perhaps already at this point, PPC receives initial projections directly from thalamic clusters, to prime the cortically incoming information. The fact that these thalamo-parietal projections are stronger during ANGLE might mean that the thalamus is primed beforehand that the incoming information is relevant to PPC, or that communication between thalamus and PPC is more effective or lasts longer during ANGLE than during COLOR.

Information from occipital cortex will proceed along the ventral and dorsal stream to frontal areas. Dorsal frontal area SFS might maintain the template of the angle that distinguished Response 1 ("small angle") from Response 2 ("large angle"). As detailed above, the MFG can use this representation to modulate PPC by means of top-down signals. Frontal region MFG thus "focuses" the PPC processing activities to the proper parts of the stimulus, presensitizing neural activity in PPC prior to stimulus onset and/or biasing neural activity after stimulus onset.

The function of the multiple loops between PPC, MFG, and OT might be an integration of region-specific processing
Figure 7. Informed neurocognitive model of visuospatial judgment. Information flows that were drawn from our data (statistically significant influences are thick arrows, influences based on individual maps or mean GCM group maps are dashed arrows). In keywords are added the proposed functional contributions of network nodes. This chart illustrates proposed neurocognitive interactions during ANGLE task trials (see main text for further explanations).

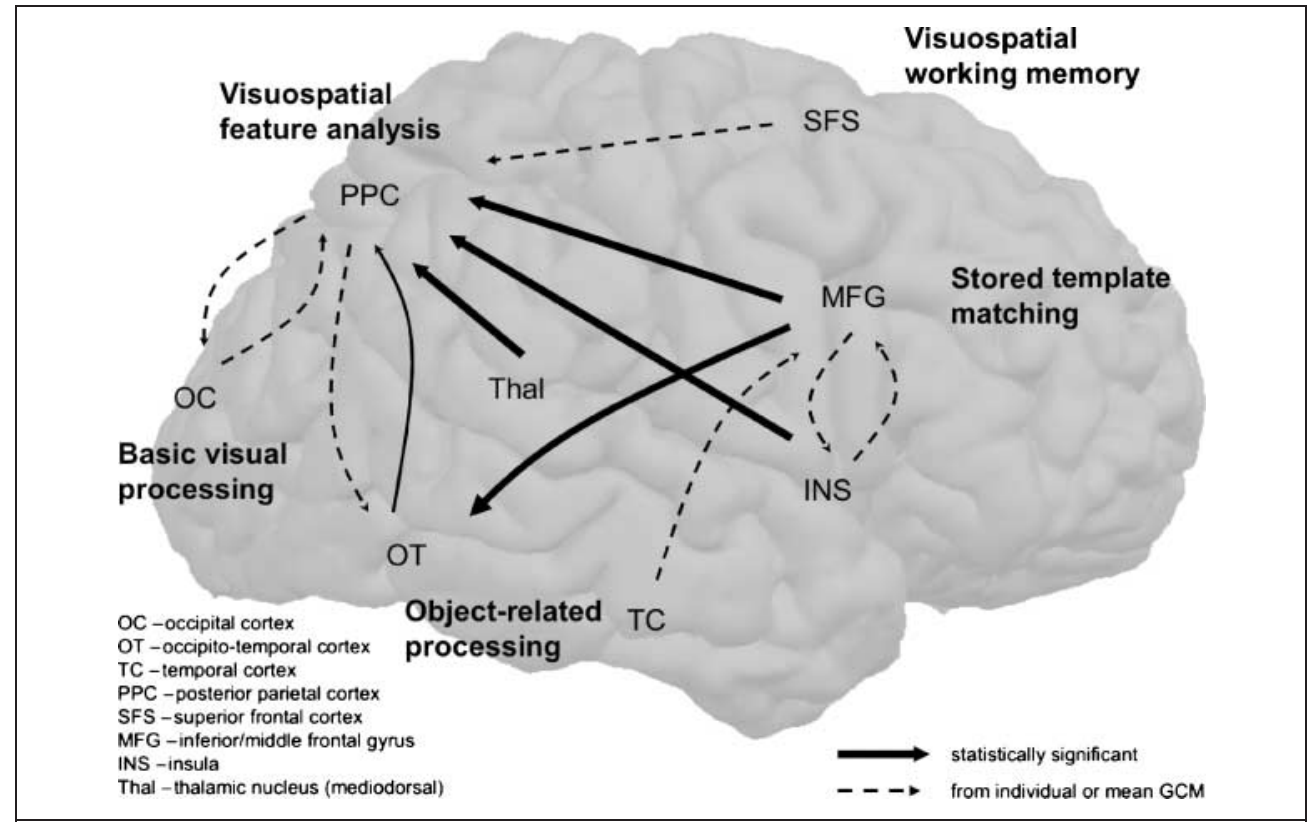


results: PPC analyzing the spatial features, MFG orchestrating which spatial features are task-relevant (on the basis of the defining visuospatial template held in SFS working memory), and OT pitching in possibly relevant object information. Such distributed processing across the brain clearly would require extensive communication, which we believe was partly revealed by our data.

Note that such interpretations necessarily remain speculative. Moreover, the model is clearly incomplete. Nevertheless, the proposed functional segregations within our network are compatible with the data as presented here and by others, and may provide fruitful new hypotheses for further research. In general, we propose that cognitive neuroscience move beyond conventional "brain mapping" to include analyses on interactions between brain areas, and to link such interactions to cognitive processes, where possible.

\section{Acknowledgments}

T. A. G. and A. T. S. were supported by grants from the Netherlands Organization for Scientific Research (NWO; grant numbers 021-002-087 and 452-06-003)

Reprint requests should be sent to Tom Alexander de Graaf, Cognitive Neuroscience, Maastricht University, P. O. Box 616 Maastricht, 6200MD, The Netherlands, or via e-mail: tom.degraaf@ maastrichtuniversity.nl.

\section{REFERENCES}

Abler, B., Roebroeck, A., Goebel, R., Hose, A., SchonfeldtLecuona, C., Hole, G., et al. (2006). Investigating directed influences between activated brain areas in a motor-response task using fMRI. Magnetic Resonance Imaging, 24, 181-185.

Banich, M. T., Milham, M. P., Atchley, R. A., Cohen, N. J., Webb, A., Wszalek, T., et al. (2000). Prefrontal regions play a predominant role in imposing an attentional "set": Evidence from fMRI. Brain Research, Cognitive Brain Research, 10, 1-9.

Behrens, T. E., Johansen-Berg, H., Woolrich, M. W., Smith, S. M., Wheeler-Kingshott, C. A., Boulby, P. A., et al. (2003). Non-invasive mapping of connections between human thalamus and cortex using diffusion imaging. Nature Neuroscience, 6, 750-757.

Bestmann, S., Thilo, K. V., Sauner, D., Siebner, H. R., \& Rothwell, J. C. (2002). Parietal magnetic stimulation delays visuomotor mental rotation at increased processing demands. Neuroimage, 17, 1512-1520.

Bien, N., Roebroeck, A., Goebel, R., \& Sack, A. T. (2009). The brain's intention to imitate: The neurobiology of intentional versus automatic imitation. Cerebral Cortex, 19, 2338-2351.

Boynton, G. M., Engel, S. A., Glover, G. H., \& Heeger, D. J. (1996). Linear systems analysis of functional magnetic resonance imaging in human V1. Journal of Neuroscience, 16, 4207-4221.

Buchsbaum, M. S., Buchsbaum, B. R., Chokron, S., Tang, C., Wei, T. C., \& Byne, W. (2006). Thalamocortical circuits: fMRI assessment of the pulvinar and medial dorsal nucleus in normal volunteers. Neuroscience Letters, 404, 282-287.

Chambers, C. D., Payne, J. M., Stokes, M. G., \& Mattingley, J. B. (2004). Fast and slow parietal pathways mediate spatial attention. Nature Neuroscience, 7, 217-218.

Cohen, M. S., Kosslyn, S. M., Breiter, H. C., DiGirolamo, G. J., Thompson, W. L., Anderson, A. K., et al. (1996). Changes in cortical activity during mental rotation. A mapping study using functional MRI. Brain, 119, 89-100.
Collette, F., \& Van der Linden, M. (2002). Brain imaging of the central executive component of working memory. Neuroscience and Biobehavioral Reviews, 26, 105-125.

Collignon, O., Davare, M., De Volder, A. G., Poirier, C., Olivier, E., \& Veraart, C. (2008). Time-course of posterior parietal and occipital cortex contribution to sound localization. Journal of Cognitive Neuroscience, 20, 1454-1463.

Corbetta, M., Kincade, J. M., \& Shulman, G. L. (2002). Neural systems for visual orienting and their relationships to spatial working memory. Journal of Cognitive Neuroscience, 14, 508-523.

Courtney, S. M., Petit, L., Maisog, J. M., Ungerleider, L. G., \& Haxby, J. V. (1998). An area specialized for spatial working memory in human frontal cortex. Science, 279, 1347-1351.

Culham, J. C., Cavina-Pratesi, C., \& Singhal, A. (2006). The role of parietal cortex in visuomotor control: What have we learned from neuroimaging? Neuropsychologia, 44, 2668-2684.

Curtis, C. E., \& D'Esposito, M. (2003). Persistent activity in the prefrontal cortex during working memory. Trends in Cognitive Sciences, 7, 415-423.

Davis, K. D., Kwan, C. L., Crawley, A. P., \& Mikulis, D. J. (1998). Functional MRI study of thalamic and cortical activations evoked by cutaneous heat, cold, and tactile stimuli. Journal of Neurophysiology, 80, 1533-1546.

de Lange, F. P., Hagoort, P., \& Toni, I. (2005). Neural topography and content of movement representations. Journal of Cognitive Neuroscience, 17, 97-112.

D’Esposito, M., Aguirre, G. K., Zarahn, E., Ballard, D., Shin, R. K., \& Lease, J. (1998). Functional MRI studies of spatial and nonspatial working memory. Brain Research, Cognitive Brain Research, 7, 1-13.

Edin, F., Klingberg, T., Stodberg, T., \& Tegner, J. (2007). Fronto-parietal connection asymmetry regulates working memory distractibility. Journal of Integrative Neuroscience, 6, 567-596.

Erickson, K. I., Ringo Ho, M. H., Colcombe, S. J., \& Kramer, A. F. (2005). A structural equation modeling analysis of attentional control: An event-related fMRI study. Brain Research, Cognitive Brain Research, 22, 349-357.

Fischl, B., Sereno, M. I., Tootell, R. B., \& Dale, A. M. (1999). High-resolution intersubject averaging and a coordinate system for the cortical surface. Human Brain Mapping, 8, 272-284.

Forman, S. D., Cohen, J. D., Fitzgerald, M., Eddy, W. F., Mintun, M. A., \& Noll, D. C. (1995). Improved assessment of significant activation in functional magnetic resonance imaging (fMRI): Use of a cluster-size threshold. Magnetic Resonance in Medicine, 33, 636-647.

Formisano, E., Linden, D. E., Di Salle, F., Trojano, L., Esposito, F., Sack, A. T., et al. (2002). Tracking the mind's image in the brain: I. Time-resolved fMRI during visuospatial mental imagery. Neuron, 35, 185-194.

Friston, K. (1994). Functional and effective connectivity in neuroimaging: A synthesis. Human Brain Mapping, 2, 56-78.

Genovese, C. R., Lazar, N. A., \& Nichols, T. (2002). Thresholding of statistical maps in functional neuroimaging using the false discovery rate. Neuroimage, 15, 870-878.

Giessing, C., Fink, G. R., Rosler, F., \& Thiel, C. M. (2007). fMRI data predict individual differences of behavioral effects of nicotine: A partial least square analysis. Journal of Cognitive Neuroscience, 19, 658-670.

Glahn, D. C., Kim, J., Cohen, M. S., Poutanen, V. P., Therman, S., Bava, S., et al. (2002). Maintenance and manipulation in spatial working memory: Dissociations in the prefrontal cortex. Neuroimage, 17, 201-213.

Goebel, R., Linden, D. E., Lanfermann, H., Zanella, F. E., \& Singer, W. (1998). Functional imaging of mirror and inverse reading reveals separate coactivated networks for oculomotion and spatial transformations. NeuroReport, 9, 713-719. 
Grill-Spector, K. (2003). The neural basis of object perception. Current Opinion in Neurobiology, 13, 159-166.

Hagler, D. J., Jr., Saygin, A. P., \& Sereno, M. I. (2006). Smoothing and cluster thresholding for cortical surface-based group analysis of fMRI data. Neuroimage, 33, 1093-1103.

Haxby, J. V., Grady, C. L., Horwitz, B., Ungerleider, L. G., Mishkin, M., Carson, R. E., et al. (1991). Dissociation of object and spatial visual processing pathways in human extrastriate cortex. Proceedings of the National Academy of Sciences, U.S.A., 88, 1621-1625.

Hilgetag, C. C., Theoret, H., \& Pascual-Leone, A. (2001). Enhanced visual spatial attention ipsilateral to rTMS-induced "virtual lesions" of human parietal cortex. Nature Neuroscience, 4, 953-957.

Husain, M., \& Nachev, P. (2007). Space and the parietal cortex. Trends in Cognitive Sciences, 11, 30-36.

Jin, Y., \& Hilgetag, C. C. (2008). Perturbation of visuospatial attention by high-frequency offline rTMS. Experimental Brain Research, 189, 121-128.

Kim, Y. H., Min, S. J., Ko, M. H., Park, J. W., Jang, S. H., \& Lee, P. K. (2005). Facilitating visuospatial attention for the contralateral hemifield by repetitive TMS on the posterior parietal cortex. Neuroscience Letters, 382, 280-285.

Klingberg, T. (2006). Development of a superior frontalintraparietal network for visuo-spatial working memory. Neuropsychologia, 44, 2171-2177.

Klingberg, T., Forssberg, H., \& Westerberg, H. (2002). Increased brain activity in frontal and parietal cortex underlies the development of visuospatial working memory capacity during childhood. Journal of Cognitive Neuroscience, 14, 1-10.

Kriegeskorte, N., \& Goebel, R. (2001). An efficient algorithm for topologically correct segmentation of the cortical sheet in anatomical MR volumes. Neuroimage, 14, 329-346.

Kveraga, K., Ghuman, A. S., \& Bar, M. (2007). Top-down predictions in the cognitive brain. Brain and Cognition, 65, 145-168.

Lamme, V. A., \& Roelfsema, P. R. (2000). The distinct modes of vision offered by feedforward and recurrent processing. Trends in Neurosciences, 23, 571-579.

Lehmann, C., Vannini, P., Wahlund, L. O., Almkvist, O., \& Dierks, T. (2006). Increased sensitivity in mapping task demand in visuospatial processing using reaction-timedependent hemodynamic response predictors in rapid event-related fMRI. Neuroimage, 31, 505-512.

Luks, T. L., Sun, F. T., Dale, C. L., Miller, W. L., \& Simpson, G. V. (2008). Transient and sustained brain activity during anticipatory visuospatial attention. NeuroReport, 19, 155-159.

Mayer, A. R., Seidenberg, M., Dorflinger, J. M., \& Rao, S. M. (2004). An event-related fMRI study of exogenous orienting: Supporting evidence for the cortical basis of inhibition of return? Journal of Cognitive Neuroscience, 16, 1262-1271.

Mellet, E., Tzourio, N., Crivello, F., Joliot, M., Denis, M., \& Mazoyer, B. (1996). Functional anatomy of spatial mental imagery generated from verbal instructions. Journal of Neuroscience, 16, 6504-6512.

Muri, R. M., Buhler, R., Heinemann, D., Mosimann, U. P., Felblinger, J., Schlaepfer, T. E., et al. (2002). Hemispheric asymmetry in visuospatial attention assessed with transcranial magnetic stimulation. Experimental Brain Research, 143, 426-430.

Owen, A. M., Evans, A. C., \& Petrides, M. (1996). Evidence for a two-stage model of spatial working memory processing within the lateral frontal cortex: A positron emission tomography study. Cerebral Cortex, 6, 31-38.

Petit, L., Courtney, S. M., Ungerleider, L. G., \& Haxby, J. V. (1998). Sustained activity in the medial wall during working memory delays. Journal of Neuroscience, 18, 9429-9437.

Petrides, M. (1994). Frontal lobes and behaviour. Current Opinion in Neurobiology, 4, 207-211.
Postle, B. R., Berger, J. S., Taich, A. M., \& D’Esposito, M. (2000). Activity in human frontal cortex associated with spatial working memory and saccadic behavior. Journal of Cognitive Neuroscience, 12(Suppl. 2), 2-14.

Roebroeck, A., Formisano, E., \& Goebel, R. (2005). Mapping directed influence over the brain using Granger causality and fMRI. Neuroimage, 25, 230-242.

Rounis, E., Yarrow, K., \& Rothwell, J. C. (2007). Effects of rTMS conditioning over the fronto-parietal network on motor versus visual attention. Journal of Cognitive Neuroscience, 19, 513-524.

Rushworth, M. F., Ellison, A., \& Walsh, V. (2001). Complementary localization and lateralization of orienting and motor attention. Nature Neuroscience, 4, 656-661.

Rypma, B., \& D'Esposito, M. (2003). A subsequent-memory effect in dorsolateral prefrontal cortex. Brain Research, Cognitive Brain Research, 16, 162-166.

Sack, A. T., Camprodon, J. A., Pascual-Leone, A., \& Goebel, R. (2005). The dynamics of interhemispheric compensatory processes in mental imagery. Science, 308, 702-704.

Sack, A. T., Hubl, D., Prvulovic, D., Formisano, E., Jandl, M., Zanella, F. E., et al. (2002). The experimental combination of rTMS and fMRI reveals the functional relevance of parietal cortex for visuospatial functions. Brain Research, Cognitive Brain Research, 13, 85-93.

Sack, A. T., Jacobs, C., De Martino, F., Staeren, N., Goebel, R., \& Formisano, E. (2008). Dynamic premotor-to-parietal interactions during spatial imagery. Journal of Neuroscience, 28, 8417-8429.

Sack, A. T., Kohler, A., Bestmann, S., Linden, D. E., Dechent, P., Goebel, R., et al. (2007). Imaging the brain activity changes underlying impaired visuospatial judgments: Simultaneous fMRI, TMS, and behavioral studies. Cerebral Cortex, 17, 2841-2852.

Sarter, M., Givens, B., \& Bruno, J. P. (2001). The cognitive neuroscience of sustained attention: Where top-down meets bottom-up. Brain Research, Brain Research Reviews, 35 , 146-160.

Scherf, K. S., Sweeney, J. A., \& Luna, B. (2006). Brain basis of developmental change in visuospatial working memory. Journal of Cognitive Neuroscience, 18, 1045-1058.

Talairach, J., \& Tournoux, P. (1988). Co-planar stereotaxic atlas of the human brain: 3-Dimensional proportional system, an approach to cerebral imaging. Stuttgart: Thieme.

Tanibuchi, I., \& Goldman-Rakic, P. S. (2003). Dissociation of spatial-, object-, and sound-coding neurons in the mediodorsal nucleus of the primate thalamus. Journal of Neurophysiology, 89, 1067-1077.

Trojano, L., Grossi, D., Linden, D. E., Formisano, E., Goebel, R., Cirillo, S., et al. (2002). Coordinate and categorical judgements in spatial imagery. An fMRI study. Neuropsychologia, 40, 1666-1674.

Trojano, L., Grossi, D., Linden, D. E., Formisano, E., Hacker, H., Zanella, F. E., et al. (2000). Matching two imagined clocks: The functional anatomy of spatial analysis in the absence of visual stimulation. Cerebral Cortex, 10, 473-481.

Valero-Cabre, A., Pascual-Leone, A., \& Rushmore, R. J. (2008). Cumulative sessions of repetitive transcranial magnetic stimulation (rTMS) build up facilitation to subsequent TMS-mediated behavioral disruptions. European Journal of Neuroscience, 27, 765-774.

Yeh, Y. Y., Kuo, B. C., \& Liu, H. L. (2007). The neural correlates of attention orienting in visuospatial working memory for detecting feature and conjunction changes. Brain Research, 1130, 146-157.

Zarahn, E., Aguirre, G. K., \& D’Esposito, M. (1999). Temporal isolation of the neural correlates of spatial mnemonic processing with fMRI. Brain Research, Cognitive Brain Research, 7, 255-268. 
Copyright of Journal of Cognitive Neuroscience is the property of MIT Press and its content may not be copied or emailed to multiple sites or posted to a listserv without the copyright holder's express written permission. However, users may print, download, or email articles for individual use. 